\title{
Síndrome de Burnout en época de pandemia: caso colombiano
}

\author{
De Arco Paternina, Lilian Karina \\ Universidad Americana de Europa (UNADE), Colombia \\ lilian.dearco@gmail.com
}

\author{
Castillo Hernández, José Andrés \\ Universidad Americana de Europa (UNADE), México \\ joseandres.castillo@aulagrupo.es
}

Resumen - En este artículo se hace una revisión de la literatura entre los años 2018 y 2020 sobre el Síndrome de Burnout (SB). Se trata de determinar qué impacto tiene éste en los profesionales colombianos (especialmente durante la pandemia actual), qué tendencias hay sobre el tema, y la contribución teórica que abordan. Primero, se contextualiza el marco de estudio con base al síndrome de burnout y su incidencia en diversas profesiones, luego, se expone la metodología que encauzó la revisión bibliográfica. El síndrome de Burnout ha sido (y aún sigue siendo) objeto de estudio en numerosas investigaciones desde hace más de tres décadas. Es así como, a través de estas, se ha observado su incidencia de forma distinta dependiendo del área de desempeño de cada profesión. De aquí la importancia de conocer en qué consiste el síndrome de burnout, síntomas y cómo las situaciones que enfrentan actualmente los profesionales del país a raíz de la pandemia hacen que se vuelva la mirada al mismo.

Palabras clave - Burnout; Estrés laboral; Salud mental; Estrés emocional; Pandemia;

\begin{abstract}
This article reviews the literature between 2018 and 2020 on Burnout Syndrome. It is about determining what impact this has on Colombian professionals (especially during the current pandemic), what trends there are on the subject, and the theoretical contribution they address. First, the study framework is contextualized based on burnout syndrome and its incidence in various professions, then the
\end{abstract}

Interconectando Saberes, 2020

ISSN: 2448-8704 methodology that guided the bibliographic review is exposed. Burnout syndrome has been (and still is) the subject of study in numerous investigations for more than three decades. This is how, through these, its incidence has been observed in a different way depending on the performance area of each profession. Hence the importance of knowing what the burnout syndrome consists of, symptoms and how the situations currently faced by professionals in the country because of the pandemic make us look back at it.

Keywords - Burnout; Work stress; Mental health; Emotional stress; Pandemic;

\section{INTRODUCCIÓN}

Actualmente, el mundo entero está afrontando una situación que se ha considerado como la "primera pandemia global de la historia" (Ribot et al. 2020 , p. 3), por cuenta del SARS-CoV-2 conocido también como COVID-19. Esto ha llevado a todos los países a adoptar medidas de aislamiento que han cambiado la forma de trabajar. En Colombia se acogieron dos modalidades de trabajo: trabajo en casa y teletrabajo. Cada una de ellas, con las particularidades que implican.

Fecha de Recepción: 10 de junio de 2020

Fecha de Aceptación: 22 de julio de 2020 Fecha de Publicación: 24 de julio de 2020 
Precisamente son estas situaciones de "teletrabajo y "trabajo en casa" (derivadas de la Emergencia sanitaria, económica y social), a las cuales se han tenido que enfrentar todos los profesionales colombianos asociadas al "encierro", al tener que ejercer labores propias del hogar, así como, el hecho de adaptarse a estas nuevas condiciones de trabajo, lo que ha provocado un aumento del síndrome de agotamiento en ellos. Como lo menciona Ramírez et al (2020), se considera a la pandemia por coronavirus 2019 como "una emergencia de salud pública de preocupación internacional, con impactos sin precedentes en el siglo XXl" (p.2) representando un gran desafío para la salud mental.

García-Campayo et al. (2016) definen el síndrome de "agotamiento emocional", "agotamiento profesional", "quemado en el trabajo" o "síndrome de burnout" (por su sigla en inglés) como el "resultado de situaciones de estrés crónico generadas por el trabajo" (p.401). De igual modo, es considerado la "enfermedad psicológica" de aquellos profesionales que trabajan con otros seres humanos (Torres et al. 2019, p108), teoría que han desvirtuado otros autores que se expondrán más adelante.

El síndrome de burnout o desgaste profesional ha sido (y aún sigue siendo) objeto de estudio en numerosas investigaciones desde hace más de tres décadas. En Colombia, ha cobrado gran importancia en la última década y más ahora que los profesionales e inclusive los estudiantes universitarios se enfrentan a esta pandemia.

\section{MetOdología}

La revisión y análisis del tema seleccionado se dividió en dos etapas: 1) Búsqueda de literatura en bases de datos para seleccionar artículos de investigación. 2) Desarrollo de una matriz de usabilidad de revisión analítica, que permita documentar y describir temas y cualidades de la investigación relacionados con el objeto de estudio. En la primera, se realizó una revisión bibliográfica de artículos, tesis de pregrado y tesis doctorales en los períodos comprendidos entre 2018 y 2020 . Se consultaron fuentes de información como bases de datos: Scopus, Ebsco, Dialnet, ScienceDirect, SciELO; así como el buscador Google académico. La estrategia de búsqueda fue a través de palabras clave, en español e inglés (burnout, agotamiento emocional, síndrome del trabajador quemado, work stress, condiciones mentales en la pandemia). Se recuperaron más de 60 citas bibliográficas de las cuales se seleccionaron 32 para el presente artículo.

Específicamente, este artículo tiene como objetivo analizar las contribuciones de los trabajos de investigación publicados entre 2018 y 2020, que aporten a la conceptualización sobre síndrome de desgaste profesional en época de pandemia. 
De conformidad a lo anterior, la pregunta que orienta esta búsqueda es: ¿Cuáles son las tendencias de investigación sobre síndrome de agotamiento emocional en los profesionales en los últimos años y su incidencia en medio de la pandemia?

\section{Nivel teórico}

El concepto de Síndrome de agotamiento emocional, desgaste profesional o burnout inició con el estudio de Herbert Freudenberger (1974), quien denominó al mismo como un estado de "fatiga mental y gradual, pérdida de motivación y compromiso hacia el trabajo" (Ibarra, et al 2018, p72). De igual modo, inició su investigación indicando que es la "descripción conceptual y operacional" presentada por un individuo a nivel psicológico, que representa un gran agotamiento emocional a largo plazo tanto a nivel de interpretación como respuesta (Torres, et al., 2019, p107).

Copca et al. (2017) manifiestan que desde 1977 se habla de síndrome de burnout "como una respuesta al estrés crónico" en el entorno laboral (p.2). Según los autores, lo más sorprendente de este síndrome es el "alcance de sus síntomas", lo cual genera actitudes negativas tanto para la organización como para el colaborador mismo.

Por otra parte, Caballero et al. (2010) establecieron que en 1976 Maslach definió este síndrome como "un estado de estrés crónico" causado por el contacto entre el personal médico y sus pacientes. Este estado provoca fatiga y alienación emocional, lo que hace que las personas se mantengan alejadas entre ellas, así como de su trabajo e inclusive que duden de la "capacidad de realizarlo" (p.132).

Posteriormente, Maslach y Jackson (1981) indicaron que el síndrome del trabajador quemado se manifiesta a partir de una "perspectiva tridimensional: "agotamiento emocional, despersonalización y realización personal" (Copca et al., 2017, p.2). Aclaran estos autores que "cuando se diagnostica el SB (síndrome de burnout), podría llegar a confundirse con otras afecciones (dentro de ellas el estrés laboral); no obstante, indican que "no hay similitud entre el síndrome de desgaste profesional y otras enfermedades".

\section{Revisión de la literatura existente}

Caballero et al. (2010), establecieron que el síndrome de agotamiento emocional inicialmente sólo involucraba personas que laboraban en el sector salud (años 80); sin embargo, en la década de los 90 la lista de profesionales se amplió incluyendo personal militar, personal administrativo, entrenadores, personal judicial, técnicos informáticos, consultores, gerentes, mandos medios, atletas y amas de casa (p.133). El síndrome de burnout se estudiaba inicialmente para las profesiones que implicaban atención y servicio, sin embargo, "se reconceptualizó para mejorar su validez y hacerla aplicable a todo tipo de ocupaciones" (García-Campayo et al., 2016, p. 401). En un trabajador "el desarrollo del síndrome de desgaste profesional es un proceso gradual, que 
se realiza de forma continua con la aparición de fases más leves y agudas". Dependiendo de las precauciones o intervención tomadas, "los síntomas pueden volverse más agudos" o estables o incluso aliviarse (Ibarra et al., 2018, p-74).

Según la investigación de Maslach (1981), el síndrome de burnout ocurre en tres aspectos o dimensiones: agotamiento emocional, despersonalización y bajo rendimiento personal (también llamado cinismo). El primero hace referencia "a la pérdida gradual de la capacidad de hacer un trabajo" y representa la dimensión personal del agotamiento (Copca et al., 2017, p.3). Máynez, et al (2017) definen esta dimensión como "la sensación de no ser capaz de dar más de uno mismo emocionalmente debido a la exposición prolongada a las demandas relacionadas con el trabajo" (p126). Estos autores indican que cuando se presenta esta dimensión, "los trabajadores se sienten extremadamente fatigados (física 0 mentalmente)" debido a una larga exposición a situaciones de gran presión laboral. De igual forma, el agotamiento emocional tiende a "afectar la salud mental y/o física de las personas", generando todo tipo de "trastornos psicopatológicos como ansiedad, conductas obsesivo-compulsivas, depresión y adicciones". En las empresas, esta dimensión "origina una reducción severa en el desempeño profesional, lo cual puede conducir a una grave disminución en el desempeño profesional, "una disminución en la calidad de la atención o los servicios", una alta tasa de rotación de personal, absentismo "e incluso el abandono del trabajo" (García-Campayo et al., 2016, p401). Asimismo, Máynez et al. (2017) sostienen que cuando los empleados cambian sus "expresiones o emociones con frecuencia o con el tiempo", pueden producirse en ellos fatiga cognitiva y recursos energéticos insuficientes (p.126). Todo ello, hace que el agotamiento emocional sea considerado como la "dimensión más crítica del síndrome de burnout" y sea la variable central para "comprender este fenómeno" (García-Campayo et al., 2016, p402).

La segunda dimensión del síndrome de desgaste profesional es la despersonalización, esta hace alusión "al contexto interpersonal del trabajador y conlleva distanciarse emocionalmente de los usuarios de sus servicios adoptando "comportamientos impersonales", fríos o endurecidos (García-Campayo et al., 2016, p402).

Por último, el cinismo hace referencia a "la falta de interés, pérdida de significado", indiferencia y alienación del individuo del trabajo (Máynez et al., 2017, p.126). De igual manera, esta dimensión se considera como una percepción de "no realizar tareas adecuadamente $y$ un sentimiento de incompetencia en el puesto asignado" (GarcíaCampayo et al., 2016, p401).

El síndrome de burnout no solamente se da en el lugar de trabajo sino también en el entorno estudiantil, especialmente los universitarios quiénes son "sometidos a grandes y diversas jornadas de aprendizaje", las cuales generan altos niveles de "ansiedad, estrés y depresión". Es precisamente durante su proceso de formación, dónde los estudiantes suelen soportar una alta 
carga de estrés. En ellos, se define el síndrome de desgaste profesional "como un estado de agotamiento físico, emocional y cognitivo" causado por la participación prolongada en un ambiente estresante (Copca et al., 2017, p. 2).

\section{BURNOUT Y ESTRÉS LABORAL}

Existe una gran diferencia entre el estrés laboral y el síndrome de agotamiento profesional, el segundo habitualmente es generado o precedido por períodos repetidos del primero. GarcíaCampayo et al. (2016) señala que debido a la importancia "del mundo del trabajo" en la vida de las personas, éste es uno de los principales factores que causan estrés.

El estrés laboral tiene grandes consecuencias no sólo para los individuos sino también para las empresas. El estrés es uno de los principales factores de riesgo de accidente cerebrovascular, dolor crónico, enfermedad cardíaca, etc. El mismo juega un rol en la aparición del síndrome del trabajador quemado "relacionado con el acoso en el lugar de trabajo (o "mobbing") y trastornos adaptativos. También afecta la calidad de vida en general" (García-Campayo et al., 2016, p 401).

La siguiente figura, muestra la situación (o Panorama) de estrés laboral en Colombia
Figura 1. Panorama del Estrés laboral en Colombia

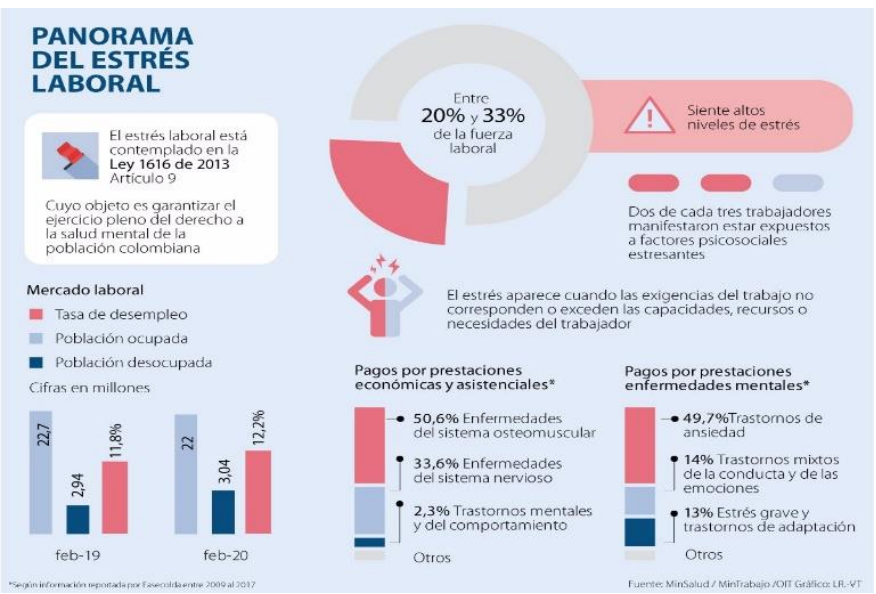

Fuente: Minsalud/MinTabajo/OIT

\section{BURNOUT EN LA PANDEMIA}

Otro punto para tener en cuenta es que, a raíz de la Pandemia, cambió la forma de laborar en Colombia. Según, FASECOLDA (Federación de Aseguradoras Colombianas) y las ARL (Administradoras de Riesgos Laborales) de "10,4 millones de trabajadores" activos en Colombia se evalúa que "2 a 3 millones se encuentran trabajando en casa" a través de dos modalidades: teletrabajo y trabajo en casa. El primero, "tiene una condición estricta" es decir, bajo el consejo de ARL, los empleadores estudian el nivel de riesgo, hacen adecuaciones físicas y proporcionan a los trabajadores de lo que necesitan: computadoras, escritorios, sillas ergonómicas. Mientras que la segunda, es una modalidad con cierta flexibilidad laboral y la cual no requiere "ningún tipo de adecuación" (según lo indicó Germán Ponce Bravo, director de la Cámara Técnica de Riesgos Laborales de FASECOLDA al diario El Tiempo). Estas nuevas modalidades de trabajar en casa son las que están generando problemas de salud y problemas mentales (los cuales se incrementan por el confinamiento y el estrés) que hacen que trabajar 
en casa sea menos ideal de lo que muchas personas imaginan, lo que conlleva a la ansiedad y la depresión. Esta nueva situación laboral en Colombia generada por la pandemia es la que ha aumentado el padecimiento del síndrome de agotamiento emocional en los profesionales de Colombia.

\section{RESULTADOS Y DISCUSIÓN}

El análisis de la información encontrada muestra que hay una gran variedad de conceptos en relación con los distintos intereses de las investigaciones sobre el tema, ya que aun cuando están fundamentadas en el síndrome de burnout y sus dimensiones, sus propósitos abarcan temas diferentes como: burnout en el ámbito académico, evolución del burnout en América Latina, burnout en estudiantes universitarios, diferencias entre burnout y otras enfermedades mentales; entre otros. Lo anterior, permite evidenciar que existen diversos marcos teóricos y conceptuales que reflejan gran diversidad en la investigación. Asimismo, se diferencian y se marcan claramente las dimensiones del burnout, así como también las situaciones laborales que han generado un aumento en el padecimiento del mismo en los profesionales del país. En el primer caso se indica que, de las 3 dimensiones del síndrome de burnout la más relevante es el agotamiento emocional; mientras que, en el segundo caso, el encierro, el cambio en las modalidades de trabajo, así como el realizar las labores del hogar, han generado una mayor propensión en los profesionales colombianos a sufrir el Síndrome del "quemado por el trabajo".
De igual forma, existen algunas estrategias para combatir el SB:

1. Identifique los factores estresantes que originan este síndrome.

2. Aprenda a administrar estos factores estresantes que conllevan al agotamiento del trabajo. Una vez que se haya identificado los factores que alimentan los síntomas de Burnout, realice un plan para resolver los problemas.

3. Cambie de actitud.

4. Realice alguna actividad física como, por ejemplo: trotar, patinar, nadar, caminar, pedalear en bicicleta, esto permite mejorar el estrés. También permite desconectarse del trabajo por unas horas.

5. Tome descansos cortos durante todo el día.

6. Ocupe parte de su tiempo practicando sus hobbies o realizando aquellas cosas que tanto le gustan.

\section{CONCLUSIONES}

Una vez realizado el análisis de artículos sobre el tema del Síndrome de desgaste profesional (burnout) en época de pandemia, es importante resaltar que este tema no sólo pertenece a un gremio de profesionales (como docentes, o profesionales del área de salud) sino que pueden padecerlo todos aquellos profesionales que se enfrenten a situaciones de estrés crónico generado por las labores diarias propias de sus cargos. 
Del mismo modo, debe tenerse en cuenta que el síndrome de burnout puede presentarse en escenarios distintos al laboral. Copca et al (2017) señala "por ejemplo en el campo estudiantil, especialmente entre los estudiantes universitarios, quiénes son sometidos a grandes y diversas jornadas de aprendizaje, con un componente alto de ansiedad, estrés y depresión" (p.2).

\section{REFERENCIAS}

Aguirre, M., C. \& Gallo, A., \& Ibarra, A., \& Sánchez G., J.C. (2018). Relación entre estrés laboral y burnout en una muestra de controladores de tráfico aéreo en Chile. Ciencias Psicológicas, 12(2), pp. 239-248. doi: https://doi.org/10.22235/cp.v12i2.1688. Recuperado de http://www.scielo.edu.uy/scielo.php?script=s ci arttext\&pid=S1688-42212018000200239.

Balayssac D, Pereira B, Virot J, Collin A, Alapini D, Cuny D, et al. (2017). Burnout, associated comorbidities and coping strategies in French community pharmacies-BOP study: A nationwide cross-sectional study. PLOS ONE 12(8). DOI:

https://doi.org/10.1371/journal.pone.0182956

Bianchi, R., \& Schonfeld, I. S. (2017). Defining Physician Burnout, and Differentiating Between Burnout and Depression-I. Mayo Clinic Proceedings, 92(9), 1455.

Bolaños, N. y Rodríguez, N. (2016). Prevalencia del Síndrome de Burnout académico en el estudiantado de Enfermería de la Universidad de Costa Rica. Rev. Enfermería Actual en Costa Rica, 31, 1-19. DOI: http://dx.doi.org/10.15517/revenf.v0i31.2451 $\underline{9}$

Caballero, D., C.C.\& Hederich, C.\& Palacio, J.E. (2010). El burnout académico: delimitación del síndrome y factores asociados con su aparición. Revista Latinoamericana de Psicología, 42 (1), pp. 131-146.
Caballero, D., C.C (2012). El burnout académico: prevalencia y factores asociados en estudiantes universitarios del área de la salud de la ciudad de Barranquilla (Tesis Doctoral). Universidad del Norte: Barranquilla, Colombia.

Caballero, D., C.C., \& Breso, É., \& González, G. Orlando (2015). Burnout en estudiantes universitarios. Revista Psicología desde el Caribe -Universidad del Norte. 32 (3), págs. 424-441. http://dx.doi.org/10.14482/psdc.32.3.6217

Camasi, G., K.M (2018). Relación entre el síndrome de burnout y clima laboral en las organizaciones: una revisión conceptual (Tesis de pregrado). Universidad San Ignacio de Loyola: Lima, Perú.

Cifuentes, A., Á.; Rivera, M., D., Vera, G., R., Murad, R., Marcela, S., S., Castaño, L.M., Royo, M., Rivillas, G. J.C. (2020). Ansiedad, depresión y miedo: impulsores de la mala salud mental durante el distanciamiento físico en Colombia, 8-20 de abril de 2020. Asociación Profamilia (11.05.2020) doi: https://doi.org/10.13140/RG.2.2.32144.6400 $\underline{2}$ Recuperado de https://profamilia.org.co/wpcontent/uploads/2020/05/Informe-3Ansiedad-depresion-y-miedo-impulsoresmala-salud-mental-durante-pandemiaEstudio-Solidaridad-Profamilia.pdf

Copca, G., A., \& Canales, H., C.A, Trejo, F., A.P, Muller, S., A., Mendoza, G., A.S, Reynoso, V., J, Ruvalcaba L., J.C (2017). El síndrome de Burnout como factor influyente en el rendimiento académico del estudiante universitario. Educación y salud Boletín Científico Instituto de Ciencias de la Salud Universidad Autónoma del Estado de Hidalgo, 5(10). https://doi.org/10.29057/icsa.v5i10.2542 
Díaz, B., F., Gómez, I.C (2016). La investigación sobre el síndrome de Burnout en Latinoamérica entre 2000 y el 2010. Revista Psicología desde el Caribe-Universidad del Norte. 33(1), pp.113-131. http://dx.doi.org/10.14482/psdc.33.1.8065 Recuperado de www.scielo.org.co/pdf/psdc/v33n1/v33n1a09 pdf.

Estrada, L., \& H.H., De la C., A., \& S.A., M. (2018). Burnout académico y su relación con el bienestar psicológico en estudiantes universitarios. Revista Espacios, 39 (15), pp. 7.

Fernández, M. A., \& Ribeiro, A. A. de A. (2020). Salud mental y estrés ocupacional en trabajadores de la salud a la primera línea de la pandemia de COVID-19. Revista Cuidarte, 11(2). https://doi.org/10.15649/cuidarte.1222 Recuperado de https://revistacuidarte.udes.edu.co/index.php /cuidarte/article/view/1222

García-Campayo J, Puebla-Guedea M, HerreraMercadal P, Daudén E (2016). Burnout Syndrome and Demotivation Among Health Care Personnel. Managing Stressful Situations: The Importance of Teamwork. Actas Dermosifiliogr. 107(5), pp. 400-406.

Gómez-Perdomo, G. E., Meneses-Higuita, A. C., \& Palacio-Montes, M. C. (2017). La satisfacción laboral y el capital psicológico: factores que influyen en el síndrome de burnout. Ansiedad y Estrés, 23(2-3), pp 7175.

https://doi.org/10.1016/j.anyes.2017.09.002

Ibarra, L., M., Erazo, M., P.A., Gallego, L., F.A. (2018). Síndrome de burnout en profesores de una institución de educación superior de Manizales-Colombia. Revista de Investigaciones UCM, 18(32), 69-83. DOI: http://dx.doi.org/10.22383/ri.v18i32.114 Recuperado de http://www.revistas.ucm.edu.co/ojs/index.ph $\mathrm{p} /$ revista/article/view/114
Ilaja, B., y Reyes, C. (2015). Burnout y estrategias de inteligencia emocional en profesores universitarios: implicaciones en la salud laboral educativa. Psicología desde EI Caribe, 33 (1), pp.31-46

Madero L., J.E. \& Ulibarri B., H.A., \& Pereyra, C., A.M., \& Paredes C., F.,E., \& Briceño, G., M.A (2013). Burnout en personal administrativo de una Institución de Educación Superior Tecnológica de Yucatán, México. Revista Electrónica de Psicología Iztacala, 16(3), pp. 741-763. www.revistas.unam.mx/index.php/repi.

Marquina-Lujan, R., \& Horna-Calderon, V. (2018). Aportes al método de investigación en el síndrome de burnout. Educación Médica. https://doi.org/10.1016/i.edumed.2018.05.00 $\underline{7}$

Máynez, G., A.I., \& Cavazos, A., J., \& y Reyes, F., G. (2017). Efecto de la violación del contrato psicológico y el agotamiento emocional sobre el cinismo del empleado. Estudios Gerenciales 33, pp. 124-131. http://dx.doi.org/10.1016/j.estger.2017.04.00 $\underline{2}$

Mérida-López, S., \& Extremera, N. (2017). Emotional intelligence and teacher burnout: A systematic review. International Journal of Educational Research, 85, 121-130. http://doi.org/10.1016/i.ijer.2017.07.006

Merino-Soto, C., \& Fernández-Arata, M. (2016). Ítem único de burnout en estudiantes de educación superior: estudio de validez de contenido. Educación Médica, 18(3), 195198.

http://doi.org/10.1016/i.edumed.2016.06.019

Ministerio TIC-Colombia (s.f. de s.f. de 2020). Teletrabajo.gov.co. Recuperado el 02 de Julio de 2020. El Teletrabajo, una modalidad laboral que crece en Colombia. https://www.teletrabajo.gov.co/622/w3article-135762.html 
Navarro-Abal, Y., Gómez-Salgado, J., LópezLópez, M., \& Climent-Rodríguez, J (2018). Organisational Justice, Burnout, and Engagement in University Students: A Comparison between Stressful Aspects of Labour and University Organisation. International Journal of Environmental Research and Public Health, 15(10), 2116. https://doi:org/10.3390/ijerph15102116

Polo, J. D., Santiago, V., Navarro, M. C. \& Alí, A. (2016). Creencias irracionales, síndrome de Burnout y adicción al trabajo en las organizaciones. Psicogente, 19(35), 148160.

http://doi.org/10.17081/psico.19.35.1215.

Psyrdellis, M., Justel, N.R. (2017). El síndrome de Burnout como factor influyente en el rendimiento académico del estudiante universitario. Anuario de Investigaciones, 24

(2) pp. 301-310. Universidad de Buenos Aires.

Ribot Reyes VC, Chang Paredes N, González Castillo AL. Efectos de la COVID-19 en la salud mental de la población. Recuperado de http://www.revhabanera.sld.cu/index.php/rha b/article/view/3307/2531

Romani, M., \& Ashkar, K. (2014). Burnout among physicians. Libyan Journal of Medicine, 9(1), 23556. https://doi.org/10.3402/lim.v9.23556

Schoeps, K., Tamarit, A., de la Barrera, U., \& González Barrón, R. (2019). Effects of emotional skills training to prevent burnout syndrome in schoolteachers. Ansiedad y Estrés.

https://doi.org/10.1016/i.anyes.2019.01.002

Torres Hernández, E.F., \& El-Sahili G., L.F., \& Gutiérrez, O., V. (2019). Análisis del burnout y sus escalas de medición. Praxis investigativa Redie. 11 (20), pp 106-124.

Vaamonde, J. D., Omar, A., \& Salessi, S. (2018). From organizational justice perceptions to turnover intentions: The mediating effects of burnout and job satisfaction. Europe's Journal of Psychology, 14(3), 554-570. https://doi.org/10.5964/ejop.v14i3.1490
Vizoso Gómez, C. M., \& Arias Gundín, O. (2016). Estresores académicos percibidos por estudiantes universitarios y su relación con el burnout y el rendimiento académicos. Anuario de Psicología, 46(2), 90-97. https://doi.org/10.1016/i.anpsic.2016.07.006 